\title{
WHAT INFORMATION DO TEENAGERS WITH IDIOPATHIC SCOLIOSIS AND THEIR FAMILIES NEED WHEN FIRST DIAGNOSED?
}

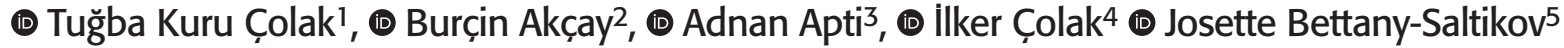 \\ ${ }_{1}^{1}$ Marmara University Faculty of Health Sciences, Department of Physiotherapy and Rehabilitation, Istanbul, Turkey \\ ${ }^{2}$ Bandırma Onyedi Eylül University Faculty of Health Sciences, Department of Physiotherapy and Rehabilitation, Balıkesir, Turkey \\ ${ }^{3}$ Istanbul Kültür University Faculty of Health Sciences, Department of Physiotherapy and Rehabilitation, Istanbul, Turkey \\ ${ }^{4}$ stanbul Kartal Dr. Lütfi Kırdar City Hospital, Clinic of Orthopaedics and Traumatology, Istanbul, Turkey \\ ${ }^{5}$ Teesside University, Department of School of Health and Social Care, Middlesbrough, United Kingdom
}

\begin{abstract}
Objective: The aim of this study was to determine the information needs of individuals with adolescent idiopathic scoliosis (AIS) and their families when the disease was first diagnosed.

Materials and Methods: A total of 117 patients with AIS and their parents volunteered to participate in this study. A survey developed specifically to assess the information needs of AIS patients and their families was sent by e-mail. Of the AIS patients, $59.8 \%$ were aged $>16$ years and completed the survey alone, and $40.2 \%$ were aged $<16$ years and they completed the survey together with their parents.

Results: The results of this study demonstrated that in the initial diagnosis, scoliosis could be observed even if the angle was $<20^{\circ}$. Generally, scoliosis was first noticed by the child's mother. As expected, at the time of the initial diagnosis, the children diagnosed as having scoliosis were upset and confused and their parents also felt upset and worried. The information most needed was reported to be answers to the questions of "Will it get better, what are the causes of scoliosis?", "What is scoliosis and what are the possible treatment options?", and the least frequently asked question was "Will surgery be needed?".

Conclusion: Clear, accurate, complete, and personalized information is required by patients and their families. This information is essential in enabling patients to make major decisions and to take ownership and responsibility for the decision. Involvement in decision-making helps to improve compliance with treatment and finally also improves satisfaction with the agreed treatment method used.

Keywords: Adolescent idiopathic scoliosis, survey, information needs, family
\end{abstract}

\section{INTRODUCTION}

Scoliosis is a three-dimensional deformity of the spine, with numerous epidemiological causes (congenital, neuromuscular, etc), and adolescent idiopathic scoliosis (AIS) is the most common (80-90\%) type ${ }^{(1-4)}$. Current treatment methods for idiopathic scoliosis include observation, physiotherapy, brace treatment and surgery ${ }^{(3-6)}$.

The appropriate treatment method is largely determined by a scoliosis orthopaedic consultant, based on a number of progression risk factors (age, maturity, curve magnitude etc.)(1). However, sometimes the decision or choice of treatment can be influenced by the consultant's belief in a particular treatment method. For example, scoliosis-specific exercise management techniques are recommended less by consultants not only in
Turkey, but also in numerous other countries, such as the UK, Australia and the USA.

However, whether the treatment process is conservative or operative, AIS patients and their families sometimes need to make major decisions. Schwieger et al.(7) reported that patients with scoliosis who did not participate in their treatment decisions (shared-decision making), reported poorer quality of life scores than those who participated in their treatment decisions. Therefore, it is essential that AIS patients and their families are fully and properly informed in making decisions related to their treatment.

Patients generally expect to be cared for by their healthcare system and to be provided with crucial and relevant information about any proposed healthcare assessment and treatment ${ }^{(8)}$. Beall et al. ${ }^{(9)}$ reported that patients with AIS and their families

Address for Correspondence: Tuğba Kuru Çolak, Marmara University Faculty of Health Sciences, Department of Physiotherapy and Rehabilitation, İstanbul, Turkey Phone: +90 5359512440 E-mail: tugba.colak@marmara.edu.tr Received: 07.05.2021 Accepted: 10.06.2021

ORCID ID: orcid.org/0000-0002-3263-2278 
turkishspine

used the internet nearly twice as much as children and teenagers diagnosed with other orthopaedic conditions in order to try and understand their diagnosis.

Previous studies that have evaluated the information needs of scoliosis patients and their families have mainly focused on the experiences of patients undergoing surgical management ${ }^{(10-13)}$. Furthermore, there is a limited number of research studies that have questioned patients about what information they need with regards to scoliosis surgery ${ }^{(14,15)}$. Rullander et al. ${ }^{(10)}$ questioned the experiences of patients and their families following scoliosis surgery, in respect of postoperative pain, and nausea, and general satisfaction both before and after the hospitalization.

In a recent study in the UK, Wellburn et al.(16) evaluated the information needs and satisfaction levels of AIS patients and their parents when they were first diagnosed. The study also explored their emotions together with the ease with which they were able to obtain information from the internet. The authors also evaluated the adequacy of the information obtained from the internet when the children were diagnosed.

Not only is little known about the information needs of AIS patients and their families, but in Turkey no studies to date have examined these information needs. Therefore, the aim of this study was to evaluate the information needs of individuals with AIS and their families at first diagnosis.

\section{MATERIALS AND METHODS}

The research proposal has been approved by Bandırma Onyedi Eylül University Faculty of Health Sciences, NonInterventional Ethics Committee with the number 2020-14 and dated 06.03.2020. The study was approved by the hospital Institutional Review Board and was conducted in compliance with the Helsinki Declaration. The informed consent was signed by both the volunteers and one of their legal representatives.

\section{Participants}

A survey was sent by e-mail to 150 individuals with AIS who were admitted to İstanbul Kartal Dr. Lütfi Kırdar City Hospital, Clinic of Orthopaedics and Traumatology between March-April 2020.

The patients included in the study were those with a diagnosis of AIS, with child and parents willing to participate in the research. The study exclusion criteria were defined as follows: Non-idiopathic scoliosis, any rheumatological or orthopedic disease, any mental disorder, or a history of spinal operation.

\section{Instrument of Data Collection}

The information needs survey in AIS is divided into two sections and consists of 18 items in total. The first 8 items are about patient characteristics that are not personally identifiable, such as age, gender, curve type, and curve size. The remaining items focused on the information needs and emotional responses of each patient and their parent. Permission for use was obtained from the developers of the questionnaire. When predefined responses were given, they were supported with free text boxes for the participants to enter alternative responses, with the instruction to choose one or all responses. A summary of the questions used in the research is presented in Appendix $1^{(16,17)}$.

\section{Data Analysis}

Data obtained in the study were analyzed statistically using IBM SPSS Statistics 21.0 (SPSS Inc, Chicago, IL,ABD). Descriptive statistics were reported as mean \pm standard deviation, minimum-maximum values for numerical variables, and as frequency and percentage for all qualitative variables.

\section{RESULTS}

A total of 117 AIS patients (96 girls, 82.10\% and 21 boys, $17.90 \%)$ volunteered to participate in this study. $59.80 \%(n=70)$ of the participants were aged $>16$ years and completed the questionnaire alone and it was completed together with the parents by the $40.20 \%(n=47)$ of AIS patients aged $<16$ years. The mean age of the patients when they first attended the hospital due to scoliosis was $13.70 \pm 3.20$ years old (range:10-18 years).

Four different curve types and five curve sizes (relative to the Cobb angle) were defined as the characteristics of scoliosis curvature. The most common curve types were thoracolumbar and lumbar curves, and the most common ranges of curve size were $10^{\circ}-19^{\circ}$ and $20^{\circ}-29^{\circ}$ (Table 1 ).

When the patient and parents were asked who was the first person to notice a problem in the teenagers back, the first people to notice a potential back problem were stated to be mother $(42.7 \%)$, doctors $(22.2 \%)$, themselves $(13.7 \%)$, family relatives $(6 \%)$, father $(5.10 \%)$, teachers $(4.30 \%)$, sisters $(2.6 \%)$, physiotherapists $(21.7 \%)$, brother $(0.9 \%)$, and school screening (0.9\%). The time taken to be referred for the first admission to hospital was reported to be $0-6$ months (20.5\%), 1 year (14.5\%), 2 years (11.1\%), 3 years $(9.4 \%)$ and $>3$ years $(44.4 \%)$.

The participants were asked to select all the options that depicted different emotions from a predetermined list of seven options. Both the patients and their parents were asked to state all the emotions they had felt when their child was diagnosed

Table 1. Curve characteristics of the study participants

\begin{tabular}{lll}
\hline Classification of curves & $\mathbf{n}$ & $\%$ \\
\hline Thoracic & 11 & 9.4 \\
\hline Lumbar & 37 & 31.6 \\
\hline Double-major & 31 & 25.5 \\
\hline Thoracolumbar & 38 & 32.5 \\
\hline Curve magnitude & & \\
\hline $\mathbf{1 0 ^ { \circ } - 1 9 ^ { \circ }}$ & 39 & 33.3 \\
\hline $\mathbf{2 0 ^ { \circ } - 2 9 ^ { \circ }}$ & 34 & 29.1 \\
\hline $\mathbf{3 0 ^ { \circ } - 3 9 ^ { \circ }}$ & 31 & 26.5 \\
\hline $\mathbf{4 0 ^ { \circ } - 4 9 ^ { \circ }}$ & 11 & 9.4 \\
\hline $\mathbf{5 0 ^ { \circ }}$ & 2 & 1.7 \\
\hline
\end{tabular}


with scoliosis. The pre-determined emotions and responses are shown in Table 2. Feelings of being upset and confused were the most frequently reported.

When the children and their parents were told that they had scoliosis, the participants were asked to indicate what questions they deemed to be most appropriate from the list of options regarding their specific information needs. "Will it get better? What causes scoliosis? What is scoliosis? What are the possible treatment options?" were the questions most frequently asked and the least needed information was "whether surgery would be needed". It was observed that more than half of all the scoliosis patients, whether completing the questionnaire on their own or with their parents, marked all the information needed items (Table 3).

It was stated by $95.7 \%$ of participants that they had received information about scoliosis. When asked about the source of this information it was said to have been obtained verbally (89.9\%), in writing (18.8\%) and electronically (36.8\%). The information was evaluated as very satisfactory by $11 \%$ of the respondents, satisfactory by $43 \%$ and neither satisfactory nor satisfactory by $26 \%$. The information was said to be sufficient by $31.6 \%$, not satisfactory by $21.4 \%$, confusing by $8.5 \%$ and $8.5 \%$ did not receive any information. Of those who did not receive any information, $90 \%$ stated that they would have preferred to receive information about their deformity.

When participants were asked what could be done to improve the information they had received, the following responses were given: The information provided needed to be written in

Table 2. Distribution of responses to pre-determined feelings

\begin{tabular}{lllllll}
\hline Information need & Patient & \multicolumn{3}{c}{ Parent/Carer } & Total \\
\hline Upset & $\mathbf{n}$ & $\mathbf{\%}$ & $\mathbf{n}$ & $\mathbf{\%}$ & $\mathbf{n}$ & \% \\
\hline Devastated & 33 & 47.1 & 29 & 61.7 & 62 & 53.0 \\
\hline Annoyed & 10 & 14.3 & 7 & 14.9 & 17 & 14.5 \\
\hline Nervous & 1 & 1.4 & 4 & 8.5 & 5 & 4.3 \\
\hline Anxious & 14 & 20.0 & 14 & 29.8 & 28 & 23.9 \\
\hline Worried & 5 & 7.1 & 11 & 23.4 & 16 & 13.7 \\
\hline Confused & 19 & 27.1 & 24 & 51.1 & 43 & 36.8 \\
\hline
\end{tabular}

Table 3. Distribution of information needs responses

\begin{tabular}{lllllllll}
\hline Information need & Patient & \multicolumn{2}{l}{ Parent/Carer } & \multicolumn{2}{l}{ Total } \\
\hline & $\mathbf{n}$ & $\mathbf{\%}$ & $\mathbf{n}$ & $\mathbf{\%}$ & $\mathbf{n}$ & \% \\
\hline What is scoliosis? & 50 & 71.4 & 33 & 70.2 & 83 & 70.9 \\
\hline What causes scoliosis? & 53 & 75.7 & 35 & 74.5 & 88 & 75.2 \\
\hline Is it hereditary? & 41 & 58.6 & 29 & 61.7 & 70 & 59.8 & 81.2 \\
\hline Will it get better? & 58 & 82.9 & 37 & 78.7 & 95 & 56.4 \\
\hline What happens now? & 40 & 57.1 & 26 & 55.3 & 66 & 67.5 \\
\hline What treatment options are there for me? & 50 & 71.4 & 29 & 61.7 & 79 & 63.8 \\
\hline Will I need an operation? & 41 & 58.6 & 22 & 46.8 & 63 & 53.8 \\
\hline How will it affect me in later life? & 46 & 65.7 & 30 & 63.8 & 76 & 65.0 \\
\hline
\end{tabular}

language that was easier to understand, it was important to increase the communication provided by doctors and it was also important to establish forum sites or websites where they could communicate with experts and each other to conduct more research and to share the results with them, and frequent public seminars could be held.

In response to the question "was this information verbal, written, or electronic?" $81.2 \%$ of participants reported that they had searched the internet for information about their diagnosis. They had searched all kinds of websites where information could be obtained, such as Google, Youtube, Pubmed, the sites of private health organizations and articles provided by healthcare professionals that provided information about scoliosis. The participants evaluated the information obtained from the internet as useful (25\%), inadequate $(23 \%)$ and confusing (17\%).

Finally, $17.9 \%$ of the participants stated that they had also received emotional support and $82.90 \%$ of them stated that it would be beneficial to communicate with people in the same situation. The respondents also commented on the ways that they would prefer to see the information prepared and presented (Table 4).

\section{DISCUSSION}

The results of this study demonstrated that at initial diagnosis, AIS can be observed even if the Cobb angle is $<20^{\circ}$. The results also showed that generally scoliosis is first noticed by mothers. 
Table 4. Participants comments

Information that participants would prefer to have

General information about scoliosis can be presented in the form of a booklet.

The musculoskeletal system can be colored to show the muscles affected by scoliosis in a way that children can understand

More detailed information about new developments could be added.

Further suggestions, and opinions regarding the information provided

The information should be given to the child, not just the parents

Not only surgical information should be given, but what should be done and what awaits patients afterwards should be explained.

Suggesting only swimming about sports that can be done restricts children a lot. More options should be offered.

When giving information, the psychology of the family and child should be given importance and the specialist should motivate the patient.

Other comments emphasizing that it was important to raise awareness and to explain all available treatment options

It should be explained that it is a treatment that requires patience

The public needs to be made aware of scoliosis by providing regular seminars.

Children's awareness of scoliosis needs to be addressed in schools

At the initial diagnosis the patients felt upset and confused and parents reported feeling very upset and worried on learning that their child had scoliosis. The following were the most common information they wanted to know: "Will it get better?" (81.2\%), "What are the causes of scoliosis?", What is scoliosis?", and "What are the possible treatment options?". The information they least wanted to know was whether or not they would need surgery (53.8\%). However, it was reported that surgeons generally mentioned this, whether surgery was needed or not. A total of 117 AIS patients participated in the study, of which $59.8 \%$ were older than 16 years and completed the survey themselves. The average age at the time of admission to the first hospital with the diagnosis of scoliosis was 13.7 years, and most of the individuals diagnosed with AIS were female (82.1\%). In a similar study in the UK, Wellburn et al.(16) reported that $58 \%$ of the patients with AIS were older than 16 years and completed the survey themselves, the mean age of the individuals was 13.3 years when first referred to the hospital and the majority were female (92\%). Furthermore, the most common curve types in the Wellburn et al. ${ }^{(16)}$ study were thoracic and lumbar, and the most common ranges of curve size were $40^{\circ}-49^{\circ}(28 \%)$ and $30^{\circ}-39^{\circ}(23 \%)$. In the current study, the most common type of curves were thoracolumbar and lumbar, and the most common curve size ranges were $10^{\circ}-19^{\circ}(33.3 \%)$ and $20^{\circ}-29^{\circ}(29.1 \%)$. Reichel and Schanz ${ }^{(18)}$ stated that the initial shock of being diagnosed with scoliosis may have resulted in emotional uncertainty together with feelings of fear, depression, despair or hopelessness that needed to be overcome. In the current study, the feelings experienced by patients and/or their parents following the diagnosis of scoliosis were observed to be mostly "feelings of being upset (53\%), confusion (39.9\%) and worry (36.8\%). In the study by Wellburn et al.(16), worry (21\%), anxiety (20\%) and being upset (19\%) were the most frequently reported feelings.

The current study findings also concur with the results of studies by Bull and Grogan ${ }^{(13)}$, van Schaik et al.(19), and Salisbury et al.(20) where patients with AIS and their parents were questioned about their feelings both before and after surgery. Confused and worried feelings, including being intensely upset were reported by the respondents. The feeling of being annoyed was the least frequent feeling and this concurred with the study of Wellburn et al.(16).

Another frequently reported feeling by the participants was the sense of feeling nervous (23.9\%). In a study by Macculloch et al. ${ }^{(14)}$, this feeling was reported before surgery. Wellburn et al. ${ }^{(16)}$ suggested that feelings of nervousness may have been caused by an "unknown fear or lack of information" following their diagnosis. Furthermore, Brosnan ${ }^{(21)}$ detailed that families with accurate and complete information reportedly had less anxiety and stress, which in turn contributed to a better overall operative experience for the patients and their parents as well as for the clinicians.

In the current study, 54\% of the participants found that the information provided about scoliosis and its treatment was satisfactory but the satisfaction levels in Turkey are generally lower than those reported by Wellburn et al.(16). As scoliosis treatment can be a long and difficult process for patients and their families, accurate knowledge with regards to any medical condition may encourage the patient to actively participate in the decision-making process and their care. Families who are informed and knowledgeable about medical conditions are then able to inform and prepare their children for the treatment processes that await them. Recent studies suggested that patients who understand their condition well are able to modify or change their decision-making behavior about treatment, and well-informed parents tend to be much more involved with their children's care and consequently were found to increase their treatment compliance ${ }^{(22-24)}$.

In the current study the vast majority of respondents (81\%) reported that they had searched the Internet about their newly diagnosed status. Wellburn et al. ${ }^{(16)}$ reported that $77 \%$ of participants had searched the Internet for information relating 
to their condition. Bull and Grogan ${ }^{(13)}$ reported that searching the Internet was the main source of information for many parents of a child who had had scoliosis surgery.

In the current study, information that the participants encountered on the Internet was found to be useful (25\%), inadequate $(23 \%)$ and confusing (17\%). Recent studies have shown that although the number of websites relating to scoliosis is high, the information on these websites is of low quality ${ }^{(25-27)}$. Regardless of health literacy, Gutierrez et al. ${ }^{(28)}$ stated that patients rely primarily on healthcare providers to obtain health information.

Therefore, it is very important that all healthcare professionals take responsibility for educating their patients and referring them to appropriate valid and reliable websites. Furthermore, it is crucial that healthcare professionals are fully informed of the content of the websites that they recommend to patients ${ }^{(25,27)}$. Wellburn et al.(25) further recommended that websites should be designed to meet AIS patient needs and that the content should be clear, evidence- based and regularly updated.

In this study conducted in Turkey, the participants stated that they primarily accessed information about scoliosis on the Internet from "Google", "Youtube", "Pubmed", and the websites of medical centers together with research papers provided by healthcare professionals.

Nason et al.(26) demonstrated that the webpages provided by academics and doctors contained higher quality information than other web pages. Nason et al. ${ }^{(26)}$ stated that as clinicians, it is not only necessary to direct patients to appropriate websites, but it is also important that healthcare professionals help in the development of high quality content on the Internet. Furthermore, nearly two decades ago, Beall et al.(9) reported that patients with scoliosis and their families used the internet to understand their diagnosis nearly twice as often as those diagnosed with other orthopedic conditions. As Internet usage is very common today it is very important that the content of websites is prepared by scoliosis professionals in their native language so as to correctly inform patients and provide them and their families with the knowledge and information that they need and which is applicable to them.

In the current study, the vast majority of participants stated that they had not received any emotional support, and they suggested that it would be beneficial to communicate with other young people in the same situation. Reichel and Schanz ${ }^{(18)}$ emphasized that support for patients is both necessary and helpful and the provision of psychological group sessions and individual discussions is likely to prevent psychosocial disorders. This is consistent with the results of a study by Hinrichsen et al. ${ }^{(29)}$, in which it was reported that most patients were very satisfied when joining a scoliosis group.

A number of valuable suggestions were made by the study participants on how they would prefer to see the information prepared and presented. These suggestions were as follows "General information about scoliosis can be presented in the form of a booklet", "The musculoskeletal system can be colored to show the muscles affected by scoliosis in a way that children can understand" and "More detailed information about new developments could be added." In a previous study, participants preferred to see "Some information may be with pictures, something a bit friendlier, sort of tailored to people our age"(16). One of the key suggestions that the participants strongly recommended was the necessity of providing scoliosis education within schools as well as increasing public awareness through public seminars. In addition, participants stated that they wanted to talk at greater length with the healthcare professionals (physicians). The children and their parents also suggested that it would be very helpful if the language used during the medical visit included less medical jargon and more use of "easy to understand" and "user-friendly" language.

Participants stated that more detailed information was needed and for this to be provided both verbally and in writing (using pictures more in the information). They also stated that it was important that the information provided is given to the child as well as to the family. Furthermore, the participants suggested that it was very important to consider the emotional and psychological needs of the child as well as their parents, for example any issues or problems with anxiety, self-confidence and self-esteem, all of which are affected by having a spinal deformity.

In line with these recommendations, we suggest that communication and the way in which information is provided is of paramount importance to this patient group and their families, so it is important that their information needs are addressed.

In the current study, the socio-economic and socio-cultural factors related to having AIS were not addressed, and this should be a component of future studies. Furthermore, although the sample size of 117 participants may be considered small in terms of a national survey, it is a relatively large sample in terms of empirical studies within the field of scoliosis. The strengths of this study that sought to ascertain the information needs of AIS patients and their parents is the first such study conducted in Turkey. The results showed that these needs do not greatly differ from the information needs expressed by patients and their parents in the UK and elsewhere.

The results of this study can significantly inform recommendations and guidelines as well as future scoliosis policies in Turkey. It can also be considered of great importance and timely to develop international recommendations and guidelines on what the minimal standards of information provided to scoliosis patients and their parents should be. This would help patients and their parents all over the world receive the same satisfactory level of information necessary for their needs, in an attempt to try and decrease the anxiety and psychological issues experienced by patients and their parents, associated with a diagnosis of AIS.

\section{CONCLUSION}

Clear, accurate, complete and personalized information is very important to patients and their families. This information is 
essential for making major decisions regarding the best and most appropriate type of treatment needed (operative or nonoperative) for the patient as well as to increase patient and parent responsibility, compliance and satisfaction with their treatment.

The key issues that need to be considered in the decision to determine the most appropriate treatment for scoliosis are the risk factors associated with curve progression.

\section{Ethics}

Ethics Committee Approval: The research proposal has been approved by Bandırma Onyedi Eylül University, Faculty of Health Sciences, Non-Interventional Ethics Committee with the number 2020-14 and dated 06.03.2020.

Informed Consent: The informed consent was signed by both the volunteers and one of their legal representatives.

\section{Authorship Contributions}

Design:T.K.Ç., J.B.S., Data Collection or Processing: I.Ç., Analysis or Interpretation: B.A., A.A., Literature Search: T.K.Ç., B.A., A.A., J.B.S., Writing: T.K.Ç., B.A., I.Ç.

Financial Disclosure: The authors declared that this study received no financial support.

Conflict of Interest: The authors have no conflicts of interest to declare.

\section{References}

1. Weiss HR, Lehnert-Schroth C, Moramarco M \& Moramarco K, Schroth therapy: advancements in conservative scoliosis treatment. Saarbruecken: Lambert Academic Publishing (LAP); 2018.

2. Weiss HR: Befundgerechte physiotherapie bei skoliose (International edition) (3th ed) Munich: Pflaum; 2010.

3. Moramarco M, Borsysov M, Ng SY, Weiss HR. Schroth's Textbook of scoliosis and other spinal deformities. Newcastle UK: Cambridge Scholars Publishing; 2020.

4. Horne JP, Flannery R, Usman S. Adolescent idiopathic scoliosis: diagnosis and management. Am Fam Physician. 2014;89:193-8.

5. Berdishevsky H, Lebel VA, Bettany-Saltikov J, Rigo M, Lebel A, Hennes A, et al. Physiotherapy scoliosis-specific exercises - a comprehensive review of seven major schools. Scoliosis Spinal Disord. 2016;11:20.

6. Bettany-Saltikov J, Parent E, Romano $M$, Villagrasa $M$, Negrini $S$. Physiotherapeutic scoliosis-specific exercises for adolescents with idiopathic scoliosis. Eur J Phys Rehabil Med. 2014;50:111-21.

7. Schwieger T, Campo S, Steuber KR, Weinstein SL, Ashida S. An exploration of information exchange by adolescents and parents participating in adolescent idiopathic scoliosis online support groups. Scoliosis Spinal Disord. 2016;11:22.

8. Leonard KJ, Casselman M, Wiljer D. Who will demand access to their personal health record? A focus on the users of health services and what they want. Healthc Q. 2008;11:92-6.

9. Beall MS, Golladay GJ, Greenfield ML, Hensinger RN, Biermann JS. Use of the Internet by pediatric orthopaedic outpatients. J Pediatr Orthop. 2002;22:261-4.

10. Rullander AC, Jonsson $H$, Lundström $M$, Lindh V. Young people's experiences with scoliosis surgery: a survey of pain, nausea, and global satisfaction. Orthop Nurs. 2013;32:327-35.

11. Rullander AC, Isberg $S$, Karling $M$, Jonsson $H$, Lindh V. Adolescents' experience with scoliosis surgery: a qualitative study. Pain Manag Nurs. 2013;14:50-9.
12. Koch KD, Buchanan R, Birch JG, Morton AA, Gatchel RJ, Browne RH. Adolescents undergoing surgery for idiopathic scoliosis: how physical and psychological characteristics relate to patient satisfaction with the cosmetic result. Spine (Phila Pa 1976). 2001;26:2119-24.

13. Bull J, Grogan S. Children having spinal surgery to correct scoliosis: a qualitative study of parents' experiences. J Health Psychol. 2010;15:299-309.

14. Macculloch R, Donaldson S, Nicholas D, et al. Towards an understanding of the information and support needs of surgical adolescent idiopathic scoliosis patients: a qualitative analysis. Scoliosis. 2009;4:12.

15. Khetani N, Donaldson S, Wright JG. What do patients and parents know about surgery for adolescent idiopathic scoliosis?: a knowledge questionnaire. Spine (Phila Pa 1976). 2008;33:E754-8. doi:10.1097/ BRS.0b013e31818579c3

16. Wellburn S, van Schaik P, Bettany-Saltikov J. The Information Needs of Adolescent Idiopathic Scoliosis Patients and Their Parents in the UK: An Online Survey. Healthcare (Basel). 2019;7:78.

17. Wellburn, S. An Investigation of the Information Needs of Adolescent Idiopathic Scoliosis Patients and their Families. Ph.D. Thesis, Teesside University, Middlesbrough, UK, 2017.

18. Reichel D, Schanz J. Developmental psychological aspects of scoliosis treatment. Pediatr Rehabil. 2003;6:221-5.

19. van Schaik P, Flynn D, van Wersch A, Ryan KA, Ferguson V. Factors important in the design of information material for scoliosis. Int J Rehabil Res. 2007;30:163-5.

20. Salisbury MH, LaMontagne LL, Hepworth JT, Cohen F. Parents' selfidentified stressors and coping strategies during adolescents' spinal surgery experiences. Clin Nurs Res. 2007;16:212-30.

21. Brosnan $\mathrm{H}$. Nursing management of the adolescent with idiopathic scoliosis. Nurs Clin North Am. 1991;26:17-31.

22. Bavarian ZMB. Parents' fear and distress during child inpatient care. Acta Medica Iranica. 2005;355-8.

23. Ullrich PF Jr, Vaccaro AR. Patient education on the internet: opportunities and pitfalls. Spine (Phila Pa 1976). 2002;27:E185-8.

24. Rahimi S, Kiaghadi A, Fallahian N. Effective factors on brace compliance in idiopathic scoliosis: a literature review. Disabil Rehabil Assist Technol. 2020;15:917-23.

25. Wellburn S, Bettany-Saltikov J, van Schaik P. An evaluation of web sites recommended by UK NHS consultants to patients with adolescent idiopathic scoliosis at the first point of diagnosis. Spine (Phila Pa 1976). 2013;38:1590-4.

26. Nason G], Baker JF, Byrne DP, Noel J, Moore D, Kiely PJ. Scoliosisspecific information on the internet: has the "information highway" led to better information provision?. Spine (Phila Pa 1976). 2012;37:E1364-9.

27. Mathur S, Shanti N, Brkaric M, Sood V, Kubeck J, Paulino C, et al. Surfing for scoliosis: the quality of information available on the Internet. Spine (Phila Pa 1976). 2005;30:2695-700.

28. Gutierrez N, Kindratt TB, Pagels P, Foster B, Gimpel NE. Health literacy, health information seeking behaviors and internet use among patients attending a private and public clinic in the same geographic area. J Community Health. 2014;39:83-9.

29. Hinrichsen GA, Revenson TA, Shinn M. Does self-help help? An empirical investigation of scoliosis peer support groups. Journal of Social Issues. 1985;41:65-87. 


\section{Appendix -1 \\ Patient Survey Questions}

1 . Who is completing this survey?

2. How old were you/your child when first referred to the hospital for scoliosis?

3. Is the person with scoliosis male/female?

4. Please enter the letters in the first half of your postcode.

5. Who first identified there may be a problem? (please do not enter their name)

6. How long is it since you/your child had the first referral appointment at the hospital?

7. What type of curve were you/your child diagnosed with?

8. How many degrees was the curve at the first referral?

9*. How would you describe your feelings on being told you/your child had scoliosis?

10*. Was a scoliosis specialist nurse present/available when you attended for your consultation?

11*. What were your specific information needs on being told that you/your child had scoliosis?

12. Did you receive any information about your (child's) condition?

12a. If you did, was this information verbal, written, or electronic (websites)?

$12 \mathrm{~b}$. Would you like to have received information about your condition?

13. If you received information, how would you rate it?

14 . Could you explain more about why you gave the information you received that rating?

15*. What in your opinion could be done to improve the information that you received?

16: Did you search the Internet for information about your (child's) condition?

17 . Did you seek emotional support?

18*. Do you have any suggestions about what would be important to include in information and leaflets to be given to scoliosis patients and their families?

*Indicates open questions with a free text box. 